\title{
Uma atualização da lista de Cladocera Cladocera (Crustacea, Branchiopoda) do Estado de Pernambuco, Brasil
}

\author{
Carlos Eduardo Aguiar Soares ${ }^{1}$ \& Lourdes Maria Abdu Elmoor-Loureiro ${ }^{2,3}$ \\ ${ }^{1}$ Laboratório de Ecologia, Universidade Católica de Brasília - UCB, \\ QS 7, Lote 1, Bloco M, n. 329, CEP 70966-700, Taguatinga, DF, Brasil \\ ${ }^{2}$ Laboratório de Zoologia, Universidade Católica de Brasília - UCB, \\ QS 7, Lote 1, Bloco M, n. 331, CEP 70966-700, Taguatinga, DF, Brasil \\ ${ }^{3}$ Autor para correspondência: Lourdes Maria Abdu Elmoor-Loureiro, e-mail: lourdes@ucb.br
}

SOARES, C.E.A. \& ELMOOR-LOUREIRO, L.M.A. An updated checklist of Cladocera (Crustacea: Branchiopoda) from Pernambuco State, Brazil. Biota Neotrop. 11(2): http://www.biotaneotropica.org.br/ v11n2/en/abstract?short-communication+bn00711022011.

Abstract: The present study aimed to identify the Cladocera species present in 15 samples of zooplankton, taken occasionally between 1981 and 2001, from the Counties of Recife, Jaboatão dos Guararapes, Paulista e Escada (state of Pernambuco, Brazil). In total, 32 species were reported; 10 of them represent first records, which increased to 51 the number of species known to occur in Pernambuco state. The highest cladoceran species richness was observed in water bodies where macrophytes were more abundant and with more samples available.

Keywords: zooplankton, cladocerans, Brazilian Northeast.

SOARES, C.E.A. \& ELMOOR-LOUREIRO, L.M.A. Uma atualização da lista de Cladocera Cladocera (Crustacea, Branchiopoda) do estado de Pernambuco, Brasil. Biota Neotrop. 11(2): http://www.biotaneotropica. org.br/v11n2/pt/abstract?short-communication+bn00711022011.

Resumo: O presente trabalho buscou identificar as espécies de Cladocera em um lote de 15 amostras de zooplâncton, coletadas ocasionalmente entre os anos de 1981 a 2001, nos municípios pernambucanos de Recife, Jaboatão dos Guararapes, Paulista e Escada. Foram observadas 32 espécies de Cladocera, sendo que 10 delas são registradas pela primeira vez em Pernambuco, elevando para 51 o número de espécies listadas para este estado. A maior riqueza de espécies de Cladocera foi observada em corpos d'água com maior presença de macrófitas e com maior número de amostras disponíveis.

Palavras-chave: zooplâncton, cladóceros, Nordeste do Brasil. 


\section{Introdução}

Os primeiros conhecimentos sobre a fauna de Cladocera (Crustacea, Branchiopoda) do estado de Pernambuco datam do final da década de 1930 e devem-se a atuação do Dr. Otto Schubart, pesquisador do Instituto Agronômico de Pesquisas (Esteves 1998). Além de investigar a fauna ictiológica da região, Schubart colecionou grande número de amostras, de diversos grupos taxonômicos, que foram enviadas para especialistas. No caso dos cladóceros, esta atividade resultou nas primeiras listas de espécies para a região Nordeste do Brasil (Brehm \& Thomsen 1936, Brehm 1937, 1938, Schubart 1938, 1942).

Considerando-se revisões taxonômicas posteriores, estima-se que este levantamento pioneiro resultou em uma lista de 36 espécies de cladóceros para Pernambuco (cf. Elmoor-Loureiro 1998).

A avaliação da numerosa lista de localidades amostradas por Schubart mostra que estão concentradas, em sua maioria, próximo à costa pernambucana, portanto, na região hidrográfica Atlântico Nordeste Oriental (Brasil 2003). O restante do estado de Pernambuco pertence à região hidrográfica do São Francisco, pouco representada neste primeiro levantamento faunístico.

Somente cerca de 50 anos depois, novos estudos fazem referência aos cladóceros de Pernambuco (Neumann-Leitão et al. 1989, Crispim \& Watanabe 2000, Elmoor-Loureiro 2004, Paranaguá et al. 2005, Almeida et al. 2009, Elmoor-Loureiro et al. 2009, Dantas et al. 2009), com o acréscimo de seis espécies à lista já existente. Também se ampliou o número de localidades amostradas dentro da região hidrográfica do São Francisco.

Mesmo com o incremento dos estudos verificado nos últimos anos, o conhecimento da biodiversidade de Cladocera de Pernambuco está longe de ser considerado adequado, havendo extensas áreas ainda não inventariadas.

Os cladóceros são importantes constituintes da fauna de ambientes lênticos, contribuindo significativamente para a dinâmica de energia nesses ecossistemas (Sa-ardrit \& Beamish 2005). São sensíveis a variações sutis do ambiente e respondem a níveis baixos de contaminantes (De Eyto et al. 2002, Ferdous \& Muktadir 2009), o que os qualifica como bons bioindicadores. Contudo, para isso, faz-se necessário o conhecimento de sua distribuição e ecologia (De Eyto et al. 2002), o que ainda não se verifica para o estado de Pernambuco ou mesmo para todo o Brasil.

Contribuindo para ampliar o conhecimento sobre a ocorrência e distribuição dos Cladocera, o presente trabalho buscou identificar as espécies presentes em um lote de amostras de zooplâncton, coletadas no estado de Pernambuco, entre os anos de 1981 e 2001.

\section{Material e Métodos}

Foram analisadas 15 amostras qualitativas de zooplâncton, provenientes de Recife e municípios próximos, coletadas de modo não sistemático, entre os anos de 1981 e 2001 (Tabela 1, Figura 1), por L.M.A. Elmoor-Loureiro.

As amostras foram coletadas com rede de plâncton (malha entre 80 e $100 \mu \mathrm{m}$ ), lançada a partir da margem; quando um banco de macrófitas achava-se presente, a rede era passada por entre as plantas, sendo amostrado, além do zooplâncton verdadeiro, exemplares da fauna associada a macrófitas. A fixação foi realizada com formalina $4 \%$.

Para identificação dos Cladocera, as amostras foram analisadas, sob microscópio estereoscópico, em sua totalidade ou, quando a densidade era elevada, em sub-amostras até que o número de espécies se estabilizasse, respeitando-se um mínimo de 50 indivíduos (adaptado de Elmoor-Loureiro 2007). A identificação em nível específico foi realizada com auxílio de microscópio óptico, com contraste de fase (Axiolab Zeiss).

\section{Resultados}

No total das amostras, 32 espécies foram registradas, sendo que destas, 10 representam primeira ocorrência para o estado de Pernambuco (Tabela 2): Pseudosida ramosa (Daday 1904), Sarsilatona serricauda (Sars 1901), Bosmina freyi De Melo \& Hebert 1994, Bosmina hagmanni Stingelin, 1904, Coronatella monacantha (Sars, 1901), Alonella clathratula Sars, 1896, Chydorus pubescens Sars, 1901, Dunhevedia colombiensis Stingelin 1913, Ephemeroporus hybridus (Daday 1905) e Notoalona sculpta (Sars 1901).

A localidade com o maior número de espécies foi o Açude de Dois Irmãos (REC2) com 18 espécies, seguido pelo Açude de Apipucos (REC1) com 15 e o Engenho de Camaçari (ESCD) com sete (Tabela 2).

A maioria das espécies observadas pertencem à Família Chydoridae (50,0\%), seguida pelos membros da Família Macrothricidae (15,6\%), típicos habitantes da zona litorânea.

\section{Discussão}

O conhecimento sobre a fauna de Cladocera no Brasil é ainda limitado (Elmoor-Loureiro 2000). Mesmo em regiões em que se verifica um maior esforço de amostragem, como em São Paulo, novos registros de ocorrência não são incomuns (por exemplo, Santos-Wisniewski et al. 2001, 2008).

Apesar de que vários corpos d'água tenham sido amostrados no estado de Pernambuco (cf. Figura 3 em Elmoor-Loureiro, 2000), certamente o inventário ainda é incompleto. Desse modo, um pequeno número de amostras analisadas no presente trabalho forneceu dez novos registros de ocorrência para o estado, elevando para 51 o número total de espécies conhecidas, o que representa um aumento de cerca de $20 \%$.

À exceção de Dunhevedia colombiensis, os novos registros de ocorrência correspondem a espécies de ampla distribuição no Brasil (Elmoor-Loureiro 1998). Já D. colombiensis, observada no Açude de Apipucos (Recife), é uma espécie que conta com apenas um registro de ocorrência no país, no estado do Rio de Janeiro (Lopes et al. 2006).

Além da inclusão de novas espécies, feita por este estudo e por diversos autores (Crispim \& Watanabe 2000, Paranaguá et al. 2005, Elmoor-Loureiro et al., 2009), a presente lista (Tabela 2) apresenta algumas diferenças em relação à previamente disponível para o estado de Pernambuco (Elmoor-Loureiro 2000). Em grande parte, trata-se de alterações taxonômicas posteriores, como é o caso de: Alona ossiani Sinev, 1998, membro neotropical do complexo Alona affinis (Sinev 1998); Anthalona verrucosa (Sars, 1901), antes citada nos gêneros Biapertura e Alona (Van Damme et al. 2011); Alonella dadayi Birge, 1910, transferida do gênero Disparalona (cf. Smirnov 1996); Karualona muelleri (Richard 1897), membro sul-americano do complexo Alona karua (Sinev \& Hollwedel 2005); Leberis davidi (Richard 1895), transferida do gênero Alona (Sinev et al. 2005); Macrothrix elegans Sars, 1901, membro neotropical do grupo Macrothrix triserialis (Dumont et al. 2002).

Macrothrix spinosa King, 1853 achava-se na lista de espécies de Pernambuco (Elmoor-Loureiro 2000). Originalmente, Brehm (1937) havia reportado a presença de $M$. squamosa. Mais tarde, esta espécie foi posta em sinonímia com M. spinosa (cf. Smirnov 1992). Contudo, as evidências de não cosmopolitismo no gênero Macrothrix recomendam tratar as duas espécies como separadas até que esta questão seja melhor investigada (Elmoor-Loureiro 2007). Os exemplares observados no presente estudo concordam com a descrição de $M$. squamosa. 


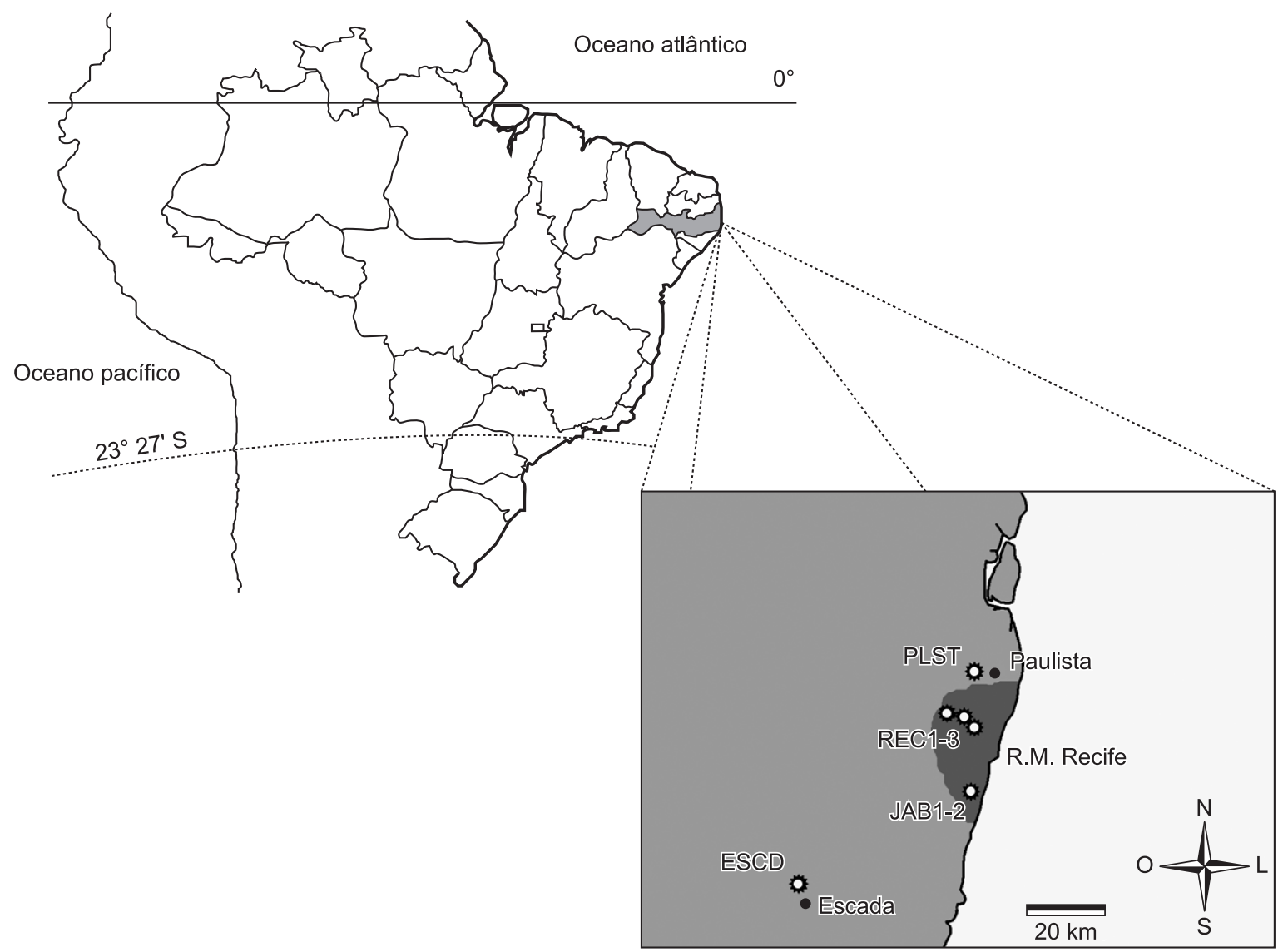

Figura 1. Localização esquemática dos corpos d'água amostrados, na região metropolitana (R.M.) de Recife e arredores. REC1-3: Município de Recife (REC1 Açude Dois Irmãos; REC2 - Açude de Apipucos; REC3 - Tanque da Praça de Casa Forte); JAB1-2: Município de Jaboatão dos Guararapes (JAB1 - Tanques do Labaratório de Oceanografia , UFPE; JAB2 - alagado próximo à praia de Piedade); ESCD: Açudes do Engenho Camaçari, Município de Escada; PLST: Lagoa do Sitio do Pica-pau Amarelo, Município de Paulista.

Figure 1. Schematic localization of the water bodies sampled in the metropolitan region of Recife (R.M. Recife) and vicinity. REC1-3: County of Recife (REC1 - Dois Irmãos reservoir; REC2 - Apipucos reservoir; REC3 - pool at Casa Forte Square); JAB1-2: County of Jaboatão dos Guararapes (JAB1 - pool at the Oceanography Laboratory, UFPE; JAB2 - swamp near Piedade Beach); ESCD: Engenho Camaçari reservoirs, County of Escada; PLST: pond at Sitio do Pica-pau Amarelo, County of Paulista.

Tabela 1. Amostras coletadas nos municípios de Recife, Jaboatão dos Guararapes, Paulista e Escada, no estado de Pernambuco. - Macrófitas ausentes; *presença de macrófitas aquáticas no local de coleta.

Table 1. Samples taken from Recife, Jaboatão dos Guararapes, Paulista, and Escada Counties, Pernambuco state. - Macrophytes absent; *macrophytes present at the sampling site.

Local de amostragem

Açude de Apipucos, Recife

Açude de Dois Irmãos, Recife

REC2

Tanque da Praça de Casa Forte, Recife

JAB1 Tanques do Lab. Oceanografia - UFPE, Jaboatão dos Guararapes

JAB2 Alagado - Praia de Piedade, Jaboatão dos Guararapes

PLST Lagoa, clube Sítio do Pica-pau Amarelo, Paulista

ESCD Engenho Camaçari, Escada (três açudes amostrados)
Coordenada

Datas

$8^{\circ} 1$ ' $16.6^{\prime \prime} \mathrm{S}$

$34^{\circ} 55^{\prime}$ 59” O

$8^{\circ} 00^{\prime} 51 " \mathrm{~S}$;

$34^{\circ} 56$ ' 47.7" O

$$
8^{\circ} 2 \text { ' 4.3" S; }
$$

$34^{\circ} 55^{\prime} 10.6$ " O

$8^{\circ} 9^{\prime} \mathrm{S}$; 34 $54^{\prime} \mathrm{O}$

$8^{\circ} 9^{\prime} \mathrm{S} ; 34^{\circ} 54^{\prime} \mathrm{O}$

$7^{\circ} 56^{\prime} \mathrm{S} ; 34^{\circ} 52^{\prime} \mathrm{O}$

$8^{\circ} 19^{\prime} \mathrm{S} ; 35^{\circ} 14^{\prime} \mathrm{O}$
$16 / 07 / 1987$

04/03/198;24/06/1991; 14/02/1998;17/01/2000;

07/09/2001

04/03/1981; 17/01/2000

(duas amostras nesta data)

04/03/1981; 24/06/1991

27/02/1981

*

$23 / 12 / 1989$
Presença

de

macrófitas ***

$-$ 
Tabela 2. Lista de espécies de Cladocera do estado de Pernambuco, com base no presente estudo (localidades indicadas como na Tabela 1) e na literatura (A - Brehm \& Thomsen 1936; B - Brehm 1937; C - Brehm 1938; D - Schubart 1938; E - Schubart 1942; F - Neumann-Leitão et al. 1989; G - Elmoor-Loureiro 1998; H - Crispim \& Watanabe 2000; I - Elmoor-Loureiro 2004; J - Paranaguá et al. 2005; K - Almeida et al. 2009; L - Elmoor-Loureiro et al. 2009; M - Dantas et al. 2009).

Table 2. Cladocera species list of Pernambuco state, based on the present study (localities indicated as in Table 1) and on literature (A - Brehm \& Thomsen 1936; B - Brehm 1937; C - Brehm 1938; D - Schubart 1938; E - Schubart 1942; F - Neumann-Leitão et al. 1989; G - Elmoor-Loureiro 1998; H - Crispim \& Watanabe 2000; I - Elmoor-Loureiro 2004; J - Paranaguá et al. 2005; K - Almeida et al. 2009; L - Elmoor-Loureiro et al. 2009; M - Dantas et al. 2009).

\begin{tabular}{|c|c|c|c|c|c|c|c|c|}
\hline & \multicolumn{7}{|c|}{ Presente estudo } & \multirow{2}{*}{ Ocorrência na literatura } \\
\hline & REC1 & REC2 & REC3 & JAB1 & JAB2 & PLST & ESCD & \\
\hline \multicolumn{9}{|l|}{ Sididae } \\
\hline Diaphanosoma brevireme Sars, 1901 & $\mathrm{x}$ & - & - & - & - & - & - & $\mathrm{B}, \mathrm{D}, \mathrm{E}, \mathrm{G}$ \\
\hline Diaphanosoma spinulosum Herbst, 1967 & $\mathrm{x}$ & - & - & - & - & - & - & $\mathrm{F}, \mathrm{G}, \mathrm{H}, \mathrm{J}, \mathrm{K}, \mathrm{M}$ \\
\hline Latonopsis australis Sars, 1888 & - & $\mathrm{x}$ & - & - & - & - & - & $\mathrm{B}, \mathrm{E}, \mathrm{G}$ \\
\hline Penilia avirostris Dana, 1852 & - & - & - & - & - & - & - & $\mathrm{J}$ \\
\hline Pseudosida ramosa (Daday, 1904) & - & $\mathrm{x}$ & - & - & - & - & - & - \\
\hline Sarsilatona serricauda (Sars, 1901) & - & - & - & - & $\mathrm{x}$ & - & - & - \\
\hline \multicolumn{9}{|l|}{ Moinidae } \\
\hline Moina micrura Kurz, 1874 & $\mathrm{x}$ & - & $\mathrm{x}$ & - & - & $\mathrm{x}$ & $\mathrm{x}$ & $\mathrm{A}, \mathrm{B}, \mathrm{C}, \mathrm{D}, \mathrm{E}, \mathrm{F}, \mathrm{G}, \mathrm{J}, \mathrm{K}, \mathrm{M}$ \\
\hline Moina minuta Hansen, 1899 & - & - & - & - & - & - & - & $\mathrm{E}, \mathrm{G}, \mathrm{H}$ \\
\hline Moinodaphnia macleayi (King, 1853) & $\mathrm{x}$ & - & - & - & - & - & $\mathrm{x}$ & $\mathrm{B}, \mathrm{C}, \mathrm{D}, \mathrm{E}, \mathrm{G}$ \\
\hline \multicolumn{9}{|l|}{ Daphniidae } \\
\hline Ceriodaphia cornuta Sars, 1886 & $\mathrm{x}$ & - & - & - & - & - & - & $\mathrm{A}, \mathrm{B}, \mathrm{C}, \mathrm{D}, \mathrm{E}, \mathrm{F}, \mathrm{G}, \mathrm{H}, \mathrm{J}, \mathrm{K}, \mathrm{M}$ \\
\hline Daphnia gessneri Herbst, 1967 & - & - & - & - & - & - & - & $\mathrm{H}, \mathrm{K}$ \\
\hline Simocephalus acutirostratus King, 1853 & - & - & - & - & - & - & - & $\mathrm{B}, \mathrm{C}, \mathrm{E}, \mathrm{G}$ \\
\hline Simocephalus latirostris Stingelin, 1906 & - & - & - & - & - & - & - & $\mathrm{B}, \mathrm{E}, \mathrm{G}$ \\
\hline Simocephalus vetulus (O.F. Müller, 1776) & - & - & - & - & - & - & - & $\mathrm{B}, \mathrm{C}, \mathrm{D}, \mathrm{E}, \mathrm{G}$ \\
\hline \multicolumn{9}{|l|}{ Bosminidae } \\
\hline Bosmina freyi De Melo \& Hebert, 1994 & $\mathrm{x}$ & - & - & - & - & - & $\mathrm{x}$ & - \\
\hline Bosmina hagmanni Stingelin, 1904 & $\mathrm{x}$ & - & - & - & - & - & $\mathrm{x}$ & - \\
\hline Bosminopsis deitersi Richard, 1895 & - & - & - & - & - & $\mathrm{x}$ & - & $\mathrm{H}$ \\
\hline \multicolumn{9}{|l|}{ Ilyocryptidae } \\
\hline Ilyocryptus spinifer Herrick, 1882 & $\mathrm{x}$ & $\mathrm{x}$ & - & $\mathrm{x}$ & - & - & - & $\mathrm{B}, \mathrm{C}, \mathrm{E}, \mathrm{G}, \mathrm{H}, \mathrm{I}, \mathrm{J}$ \\
\hline \multicolumn{9}{|l|}{ Macrothricidae } \\
\hline Grimaldina brazzai Richard, 1892 & - & - & - & - & $\mathrm{x}$ & - & - & $\mathrm{B}, \mathrm{E}, \mathrm{G}, \mathrm{H}$ \\
\hline Macrothrix elegans Sars, 1901 & - & $\mathrm{x}$ & - & - & $\mathrm{x}$ & - & - & $\mathrm{B}, \mathrm{C}, \mathrm{D}, \mathrm{E}, \mathrm{G}$ \\
\hline Macrothrix paulensis (Sars, 1900) & - & $\mathrm{x}$ & - & - & - & - & - & $\mathrm{E}, \mathrm{G}$ \\
\hline Macrothrix squamosa Sars, 1901 & - & $\mathrm{x}$ & - & - & - & - & - & $\mathrm{B}, \mathrm{D}, \mathrm{E}, \mathrm{G}, \mathrm{H}$ \\
\hline Streblocerus sp & - & - & - & - & - & - & - & $\mathrm{H}$ \\
\hline \multicolumn{9}{|l|}{ Chydoridae } \\
\hline Alona glabra Sars, 1901 & $\mathrm{x}$ & - & - & $\mathrm{x}$ & - & $\mathrm{x}$ & - & $\mathrm{A}, \mathrm{D}, \mathrm{E}, \mathrm{G}$ \\
\hline Alona ossiani Sinev, 1998 & - & - & - & - & - & - & - & $\mathrm{B}, \mathrm{E}, \mathrm{G}$ \\
\hline Alona poppei Richard, 1897 & - & $\mathrm{x}$ & $\mathrm{x}$ & - & - & - & - & $\mathrm{B}, \mathrm{E}, \mathrm{G}$ \\
\hline Anthalona verrucosa (Sars, 1901) & - & $\mathrm{x}$ & - & - & - & - & $\mathrm{x}$ & $\mathrm{B}, \mathrm{C}, \mathrm{D}, \mathrm{E}, \mathrm{G}, \mathrm{H}$ \\
\hline Alonella clathratula Sars, 1896 & - & $\mathrm{x}$ & - & - & - & - & - & - \\
\hline Alonella dadayi Birge, 1910 & - & $\mathrm{x}$ & - & - & - & - & - & $\mathrm{A}, \mathrm{B}, \mathrm{C}, \mathrm{E}, \mathrm{G}$ \\
\hline Chydorus eurynotus Sars, 1901 & - & - & - & - & - & - & - & $\mathrm{A}, \mathrm{B}, \mathrm{C}, \mathrm{D}, \mathrm{E}, \mathrm{G}, \mathrm{H}$ \\
\hline Chydorus pubescens Sars, 1901 & - & $\mathrm{x}$ & - & $\mathrm{x}$ & $\mathrm{x}$ & - & - & - \\
\hline Chydorus cf. sphaericus & - & - & - & - & - & - & - & C, E, G \\
\hline Coronatella monacantha (Sars, 1901) & - & $\mathrm{x}$ & - & - & - & - & $\mathrm{x}$ & - \\
\hline Dadaya macrops (Daday, 1898) & - & - & - & - & - & - & - & C, E, G \\
\hline Disparalona hamata (Birge, 1879) & - & - & - & - & - & - & - & $\mathrm{B}, \mathrm{E}, \mathrm{G}$ \\
\hline Dunhevedia colombiensis Stingelin, 1913 & $\mathrm{x}$ & - & - & - & - & - & - & - \\
\hline Dunhevedia odontoplax Sars, 1901 & - & $\mathrm{x}$ & - & - & - & - & - & $\mathrm{C}, \mathrm{E}, \mathrm{G}$ \\
\hline Ephemeroporus barroisi (Richard, 1894) & - & - & - & - & - & - & - & $\mathrm{J}$ \\
\hline Ephemeroporus hybridus (Daday, 1905) & - & $\mathrm{x}$ & - & - & - & - & - & - \\
\hline Euryalona brasiliensis Brehm \& Thonsem, 1936 & $\mathrm{x}$ & - & - & - & - & - & - & $\mathrm{A}, \mathrm{D}, \mathrm{D}, \mathrm{E}, \mathrm{G}, \mathrm{M}$ \\
\hline
\end{tabular}


Tabela 2. Continuação...

\begin{tabular}{|c|c|c|c|c|c|c|c|c|}
\hline & \multicolumn{7}{|c|}{ Presente estudo } & \multirow{2}{*}{ Ocorrência na literatura } \\
\hline & REC1 & REC2 & REC3 & JAB1 & JAB2 & PLST & ESCD & \\
\hline Euryalona orientalis (Daday, 1898) & - & - & - & - & - & - & - & $\mathrm{E}, \mathrm{G}$ \\
\hline Karualona mulleri (Richard, 1897) & $\mathrm{x}$ & $\mathrm{x}$ & - & - & - & - & - & $\mathrm{B}, \mathrm{C}, \mathrm{E}, \mathrm{G}, \mathrm{H}$ \\
\hline Leberis davidi (Richard, 1895) & - & - & - & - & - & - & - & $\mathrm{A}, \mathrm{B}, \mathrm{C}, \mathrm{D}, \mathrm{E}, \mathrm{G}$ \\
\hline Leydigia ipojucae Brehm, 1938 & - & - & - & - & - & - & - & $\mathrm{C}, \mathrm{E}, \mathrm{G}$ \\
\hline Leydigia schubarti Brehm \& Thonsem, 1936 & - & - & - & - & - & - & - & $\mathrm{A}, \mathrm{B}, \mathrm{C}, \mathrm{E}, \mathrm{G}$ \\
\hline Leydigiopsis brevirostris Brehm, 1938 & - & - & - & - & - & - & - & $\mathrm{E}, \mathrm{G}$ \\
\hline Leydigiopsis curvirostris Sars, 1901 & $\mathrm{x}$ & $\mathrm{x}$ & - & - & - & - & - & $\mathrm{A}, \mathrm{D}, \mathrm{E}, \mathrm{G}, \mathrm{H}$ \\
\hline Leydigiopsis ornata Daday, 1905 & - & - & - & - & - & - & - & $\mathrm{B}, \mathrm{E}, \mathrm{G}$ \\
\hline Notoalona sculpta (Sars, 1901) & - & $\mathrm{x}$ & - & - & - & - & - & - \\
\hline Oxyurella longicaudis (Birge, 1910) & $\mathrm{x}$ & $\mathrm{x}$ & - & - & - & - & $\mathrm{x}$ & $\mathrm{A}, \mathrm{B}, \mathrm{C}, \mathrm{E}, \mathrm{G}, \mathrm{H}$ \\
\hline Parvalona parva (Daday, 1905) & $\mathrm{x}$ & - & - & - & - & - & - & $\mathrm{L}$ \\
\hline
\end{tabular}

Uma outra diferença em relação à lista de espécies anterior está na substituição de Alona cambouei Guerne \& Richard 1893 e Alona pulchella King, 1853 por Alona glabra Sars 1901. A revisão deste grupo de espécies (Sinev 2001a, 2001b) mostrou que elas ocorrem em diferentes continentes e que as diferenças entre elas estão em características sutis; no Brasil, a espécie presente é A. glabra.

Os resultados do presente trabalho mostraram maior número de espécies nos Açudes de Apipucos e Dois Irmãos, o que decorre, em parte, pelo maior número de amostras analisadas para estas localidades (cinco e três, respectivamente - Tabela 1). Outro fator que pode ter contribuído para maior riqueza é a heterogeneidade espacial gerada pela maior presença de macrófitas aquáticas nestes dois açudes. A vegetação litorânea, proporciona grande diversidade de habitats (Nogueira et al. 2003), disponibilizando condições e recursos variados para a colonização por um maior número de espécies.

A composição de espécies observada nestes corpos d'água corrobora a idéia de que a presença do banco de macrófitas teve efeito positivo no aumento da riqueza. De fato, verifica-se que a maior parte das espécies registradas são típicos membros da fauna associada a macrófitas, como é o caso de Latonopsis autralis, Pseudosida ramosa, Moinodaphnia macleayi, Ilyocryptus spinifer (Sousa et al. 2009) e das espécies de Chydoridae e Macrothricidae (Elmoor-Loureiro 2007).

\section{Agradecimentos}

Os autores agradecem à Universidade Católica de Brasilia e ao Conselho Nacional de Desenvolvimento Científico e Tecnológico (projeto 555288/2006-4) pelo suporte financeiro.

\section{Referências Bibliográficas}

ALMEIDA, V.L.S., DANTAS, E.W., MELO-JÚNIOR, M., BITTENCOURTOLIVEIRA, M.C. \& MOURA, A.N. 2009. Zooplanktonic community of six reservoirs in northeast Brazil. Braz. J. Biol. 69(1): 57-65. http://dx.doi.org/10.1590/S1519-69842009000100007

BRASIL. Ministério do Meio Ambiente. Conselho Nacional De Recursos Hídricos - CNRH. 2003. Divisão Hidrográfica Nacional. Resolução $\mathrm{n}^{\circ}$ 32. http://www.cnrh-srh.gov.br/delibera/resolucoes/R032.htm (último acesso em 07/01/2010).

BREHM, V. 1937. Brasilianische Cladoceren gesammelt von Dr. O. Schubart. Zweiter Bericht. Int. Rev. Gesamten. Hydrobiol. Hydrogr. 35:497-512.

BREHM, V. 1938. Dritter Bericht über die von Dr. O. Schubart in Brasilien gesammelten Onychura. Zool. Anz. 122:94-103.

BREHM, V. \& THOMSEN, R. 1936. Brasilianische Phyllopoden und Arguliden gesammelt von Herrn Dr. O. Schubart. Zool. Anz. 116:211-218.
CRISPIM, M.C. \& WATANABE, T. 2000. Caracterização limnológica das bacias doadoras e receptoras de águas do Rio São Francisco: 1 - Zooplâncton. Acta limnol. bras. 12:93-103.

DANTAS, E.W., ALMEIDA, V.L.S., BARBOSA, J.E.L., BITTENCOURTOLIVEIRA, M.C. \& MOURA, A.N. 2009. Efeito das variáveis abióticas e do fitoplâncton sobre a comunidade zooplanctônica em um reservatório do Nordeste brasileiro. Iheringia, Ser. Zool. 99(2):132-141.

DE EYTO, E., IRVINE, K. \& FREE, G. 2002. The use of members of the Family Chydoridae (Anomopoda, Branchiopoda) as an indicator of lake ecological quality in Ireland. Biol. Environ. 102B:81-91.

DUMONT, H.J., SILVA-BRIANO, M. \& SUBHASH BABU, K.K. 2002. A re-evaluation of the Macrothrix rosea-triserialis group, with the description of two new species (Crustacea: Anomopoda: Macrothricidae). Hydrobiologia 467:1-44. http://dx.doi.org/10.1023/A:1014933227259

ELMOOR-LOUREIRO, L.M.A. 1998. Branhiopoda. Freshwater Cladocera. In Catalogue of Crustacea of Brazil (P.S. Young, ed.). Museu Nacional, Rio de Janeiro, p.15-41.

ELMOOR-LOUREIRO, L.M.A. 2000. Brazilian cladoceran studies: where do we stand? Náuplius 8:117-131.

ELMOOR-LOUREIRO, L.M.A. 2004. Morphological abnormalities in the cladoceran Ilyocryptus spinifer (Apipucos reservoir, Pernambuco state, Brazil). Braz. J. Biol. 64:53-58. http://dx.doi.org/10.1590/S1519-69842004000100007

ELMOOR-LOUREIRO, L.M.A. 2007. Phytophilous cladocerans (Crustacea, Anomopoda and Ctenopoda) from Paraná River Valley, Goiás, Brasil. Rev. Bras. Zool. 24(2):344-352. http://dx.doi.org/10.1590/S0101-81752007000200012

ELMOOR-LOUREIRO, L. M. A., SANTOS-WISNIEWSKI, M. J. \& ROCHA, O. 2009. New records of Parvalona parva (Daday, 1905) (Crustacea, Anomopoda, Chydoridae) from Brazil, with description of the male. Rev. Bras. Zool. 26:369-373.

ESTEVES, F.A. 1998. Fundamentos de Limnologia. Interciência, Rio de Janeiro.

FERDOUS, Z. \& MUKTADIR, A.K.M. 2009. A Review: Potentiality of Zooplankton as Bioindicator. Am. J. Appl. Sci. 6(10):1815-1819.

LOPES, P.M., ELMOOR-LOUREIRO, L.M.A. \& BOZELLI, R.L. 2006. First record of Dunhevedia colombiensis Stingelin, 1913 (Crustacea, Anomopoda, Chydoridae) from Brazil. Braz. J. Biol. 66(4):1141-1142. http://dx.doi.org/10.1590/S1519-69842006000600023

NEUMANN-LEITÃO, S., PARANHOS, J.D.N. \& DE SOUZA, F.B.V.A. 1989. Zooplâncton do Açude de Apipucos, Recife, PE (Brasil). Arq. Biol. Tecnol. 32:803-821.

NOGUEIRA, M.G., GEORGE, D.G. \& JORCIN, A. 2003. Estudo do zooplâncton em zonas litorâneas lacustres: um enfoque metodológico. In Ecótonos nas interfaces dos Ecossistemas Aquáticos (R. Henry, ed.). Editora Rima, São Carlos, p.83-127. 
PARANAGUÁ, M.N., NEUMANN-LEITÃO, S., NOGUEIRA-PARANHOS, J.D., SILVA, T.A. \& MATSUMURA-TUNDISI, T. 2005. Cladocerans (Branchiopoda) of a tropical estuary in Brazil. Braz. J. Biol. 65(1):107-115. http://dx.doi.org/10.1590/S1519-69842005000100015

SA-ARDRIT, P. \& BEAMISH, F.W.H. 2005. Cladocera, diversity, abundance and habitat in a Western Thailand stream. Aquat. Ecol. 39:353-365.

SANTOS-WISNIEWSKI, M.J., ROCHA, O. \& MATSUMURATUNDISI, T. 2001. First record of Alona setigera Brehm (Cladocera, Chydoridae) in the Neotropical Region. Braz. J. Biol. 61(4):701-702. http://dx.doi.org/10.1590/S1519-69842001000400022

SANTOS-WISNIEWSKI, M.J., ROCHA, O., GUNTZEL, A.M. \& MATSUMURA-TUNDISI, T. 2008. Species richness and geographic distribution of the genera Chydorus and Pseudochydorus (Cladocera, Chydoridae) in São Paulo State. Biota Neotrop. 8(1):21-23.

SCHUBART, O. 1938. Considerações sobre as investigações nas águas de Pernambuco. Arch. Inst. Pesqui. Agron. Pernamb. 1:26-57.

SCHUBART, O. 1942. Fauna do Estado de Pernambuco e estados limítrofes: segunda lista. Bol. Mus. Nac., Zool. 14/17:21-64.

SINEV, A.Y. \& HOLLWEDEL, W. 2005. Translocation of Alona muelleri Richard, 1897 into the genus Karualona Dumont \& Silva-Briano, 2000 (Branchiopoda: Anomopoda: Chydoridae). Arthropoda Selecta 14(2):93-101
SINEV, A.Y. 1998. Alona ossiani sp. n., a new species of the Alona affinis complex from Brazil, deriving from the collection of G.O. Sars (Anomopoda: Chydoridae). Arthropoda Selecta 7(2):103-110.

SINEV, A.Y. 2001a. Separation of Alona cambouei Guerne \& Richard, 1893 from Alona pulchella King, 1853 (Branchiopoda: Anomopoda: Chydoridae). Arthropoda Selecta 10(1):5-18.

SINEV, A.Y. 2001b. Redescription of Alona glabra Sars, 1901, a South American species of the pulchella-group (Branchiopoda: Anomopoda: Chydoridae). Arthropoda Selecta 10(4):273-280.

SINEV, A.Y., VAN DAMME, K. \& KOTOV, A.A. 2005. Redescription of tropical-temperate cladocerans Alona diaphana King, 1853 and Alona davidi Richard, 1895 and their translocation to Leberis Smirnov, 1989 (Branchiopoda: Anomopoda: Chydoridae). Arthropoda Selecta 14(3):183-205.

SMIRNOV, N.N. 1992. The Macrothricidae of the world. SPB Academic Publishing, Amsterdam.

SMIRNOV, N.N. 1996. Cladocera: The Chydorinae and Sayciinae (Chydoridae) of the world. SPB Academic Publishing, Amsterdam.

VAN DAMME, K., SINEV, A.Y. \& DUMONT, H.J. 2011. Separation of Anthalona gen.n. from Alona Baird, 1843 (Branchiopoda: Cladocera: Anomopoda): morphology and evolution of scraping stenothermic alonines. Zootaxa 2875: 1-64.

Recebido em 20/01/2010

Versão reformulada recebida em 06/02/2010

Publicado em 14/04/2011 